\title{
Visual Grouping and Its Application to Road Design and Traffic Control
}

\author{
Gerald Forbes ${ }^{a}$ \\ ${ }^{a}$ Intus Road Safety Engineering Incorporated, 2606 Bluffs Way, Burlington, Ontario, L7M 0T8, CANADA
}

\begin{abstract}
Visual or perceptual grouping refers to the tendency of the visual system to aggregate discrete stimuli into larger wholes. It is the process of determining which regions and parts of the visual scene belong together as parts of higher order perceptual units such as objects or patterns. The central hypothesis of Gestalt psychology is that the mind forms these global wholes through autonomous processes in the brain using the following principles - simplicity, proximity, similarity, closure, common fate, continuity, and figure-ground. An understanding of the Gestalt principles of visual grouping helps explain why alert and attentive motorists can sometimes make inexplicably bad decisions concerning speed and/or path of travel, and can be used by designers to engineer safer roads.
\end{abstract}

KEYWORDS: Visual grouping; Gestalt; road safety; highway design; traffic control

\section{INTRODUCTION}

In the vast majority of crashes, over $90 \%$, human error is identified as a contributing factor $[1,2,3]$. While it is acknowledged that a sizeable portion of these human-error crashes results from inattention and/or distraction (i.e., recognition errors), a large majority are attributed to decision, perceptual, and/ or performance errors (e.g., looked-but-failed-to-see, improper speed), and other poor driving decisions made by otherwise attentive motorists [3]. When one considers that motorists receive about $90 \%$ of the information they use to make driving decisions from the visual channel $[4,5,6]$, and that motorists most often look to the highway itself for information $[7,8]$, a logical inference is that perhaps the road scene/design is contributing to these driver errors, and many human error crashes are actually precipitated by an inadequate or confusing road design. This is known in other fields as design-induced errors [9].

Roadways around the globe are designed by engineers using national highway design guidelines and manuals $[10,11,12]$. The old paradigm of road design was based on a unifying design speed which was used in the thoughtful selection of road elements and dimensions from the prevailing design guide to produce a safe and passable road for all road users exercising reasonable care. This paradigm is supported by the design manuals themselves, which are typically structured to deliver information/guidance to the reader on individual elements of the highway with only some consideration given to how the individual elements combine, and the impact the composite visual scene has on driver behaviour. The shortcoming of this approach is that, on occasion the combination of different road elements and/or a certain road design in a certain setting creates a visual scene that results in improper driving decisions and undue crash risk. This is changing somewhat with more widespread acceptance of human factors in highway engineering $[13,14,15]$.

While the aforementioned guidelines and principles concerning the human factors of highway design address issues such as user expectancy, attention, task analysis during curve driving, etc., highway design professionals have not been imbued with the basic understanding of some general principles of visual perception that could enhance their designs. In particular, there is a paucity of discussion concerning visual grouping (also known as perceptual organization).

The purpose of this paper is to introduce the reader to the Gestalt principles of visual grouping and to demonstrate how these principles may be used in the delivery of safer road infrastructure. The principles are further illustrated through real-world examples demonstrating how ignoring the princi- 
ples can result in crashes, and conversely, how good Gestalt can promote safe and orderly movement. This paper results from the author's experience in conducting road safety audits and forensic crash investigations. An understanding of the Gestalt principles of visual grouping helps explain why alert and attentive motorists can sometimes make inexplicably bad decisions concerning speed and/or path of travel, and can be used by designers to engineer safer roads.

\section{WHAT IS VISUAL GROUPING?}

\subsection{Gestalt psychology}

Gestalt psychology is the study of how individuals integrate and organize perceptual information into meaningful wholes. In fact, Gestalt is the German word for form or shape. Gestalt psychology is a school of thought that was mainly developed at the Berlin School of experimental psychology in the early part of the last century, and attempts to explain how individuals acquire and maintain meaningful visual perceptions in an overwhelming and confusing world $[16,17]$.

The central hypothesis of Gestalt psychology is that the mind forms a global whole through selforganizing tendencies. In other words, people do not experience individual sensations and separate elements of the visual scene, rather it is structured wholes or Gestalten that are the primary units of visual input. These Gestalten are formed by autonomous processes in the brain and is reflected in the well-known saying -- the whole is other than the sum of its parts [18].

Gestalt psychology includes a number of principles of perception that determine how objects are visually grouped to form wholes or scenes. These principles of visual grouping, which are often referred to as laws or axioms of perception are more akin to heuristics.

Gestalt psychology is in direct contrast to the constructivism approach to perception, which purports that the eyes are constantly in motion while scanning an image or scene, and that individuals assemble data from a number of eye fixations to make sense of the visual scene. In other words, under constructivism, a scene is pieced together through visual information gathered by rapid eye movements, the scene is compared to memory and past associations, and an image is constructed.

\subsection{Visual grouping}

The eyes are used to gather an abundance of information about the world that surrounds us. However, the images collected by the eyes are simply a vast collection of colours, shapes, and patterns until the information is interpreted by the brain. In other words, visual information by itself is uninformative. It is only after this information is processed by the brain into visual precepts and coherent images that visual information is meaningful to the observer.

One of the cognitive techniques that individuals use to make sense of the visual world is visual grouping. Visual or perceptual grouping refers to the tendency of the visual system to aggregate discrete stimuli into larger wholes. It is the process of determining which regions and parts of the visual scene belong together as parts of higher order perceptual units such as objects or patterns. For example, a skyscraper is a complex array of light and shadow, blues and grays, and glass and metal, and while it may be perceived as a myriad of windows, columns, beams, and other objects it is more likely perceived as a single building.

Visual grouping helps individuals make sense of the world. It organizes or distills the thousands of visual signals being received by the observer into a whole or scene that is different and more meaningful than the sum of the parts.

Visual grouping, also known as perceptual organization, has two primary organizational principles - likelihood and simplicity. Likelihood is the organization of visual inputs to achieve the most likely interpretation that matches the distal stimulation. Simplicity organizes visual inputs to achieve the simplest or most economical interpretation of the stimulus. The Gestalt rules of visual grouping are predicated on the simplicity principle, which Gestalt theorists term the Law of Pragnanz. These rules can be combined with other perception principles to assist in developing safer, self-explaining roads $[19,20,21]$.

\subsection{The principles of visual grouping}

When motorists look at the road ahead they tend to see a scene that consists of a group of selected objects on a background. They do not see a vast collection of individual elements, they see a whole. The tendency for humans to organize visual inputs into organized groups and patterns is an automatic cognitive process which is reflexively activated by visual stimulation. 
This visual grouping does not require the observer to adopt a certain perceptual stance or disposition, it is a preattentive process.

Experimental psychologists postulate that visual grouping is governed by the law of Prägnanz, which asserts that individuals organize/process visual inputs in a manner that is regular, orderly, symmetrical, and simple. This law is coupled with a set of principles that, hypothetically, allow one to predict how visual scenes will be interpreted. These principles are usually referred to as the Gestalt rules, which define how individuals organize visual inputs/scenes into groups or unified wholes.

The first law of visual grouping, as previously mentioned, is the general law of Prägnanz which stipulates that the mind has a tendency towards organizing the visual scene and the objects within it in the simplest and most impressive structure given the prevailing conditions. This law, along with the following laws of visual grouping, make up the Gestalt rules of perceptual grouping:

1. Proximity - Objects that are physically close to each other form a group.

2. Similarity-Objects that are similar to each other in shape, colour, shading or other qualities are viewed as a group.

3. Closure - Individuals perceive objects as being whole when they are not quite complete. Specifically, when parts of a whole picture are missing, perception fills in the visual gap by visually grouping the individual elements.

4. Common Fate - The law of common fate implies the grouping together of objects that have the same trend of motion and are therefore on the same path.

5. Continuity - Elements are grouped together if they are aligned with each other. Elements that are connected but have sharp or abrupt directional changes are more likely to be perceived as distinct objects than as a single element.

6. Figure ground-The process of separating figures or objects from their background. Visual cues that help distinguish figures from backgrounds include size (smaller regions are often (but not always) figures), object shape (figures tend to be convex), movement (figure may be moving against a static environment), and colour (background tends to continue as one colour behind potentially multiple foreground figures).
The principles of visual grouping are illustrated in Figure 1.

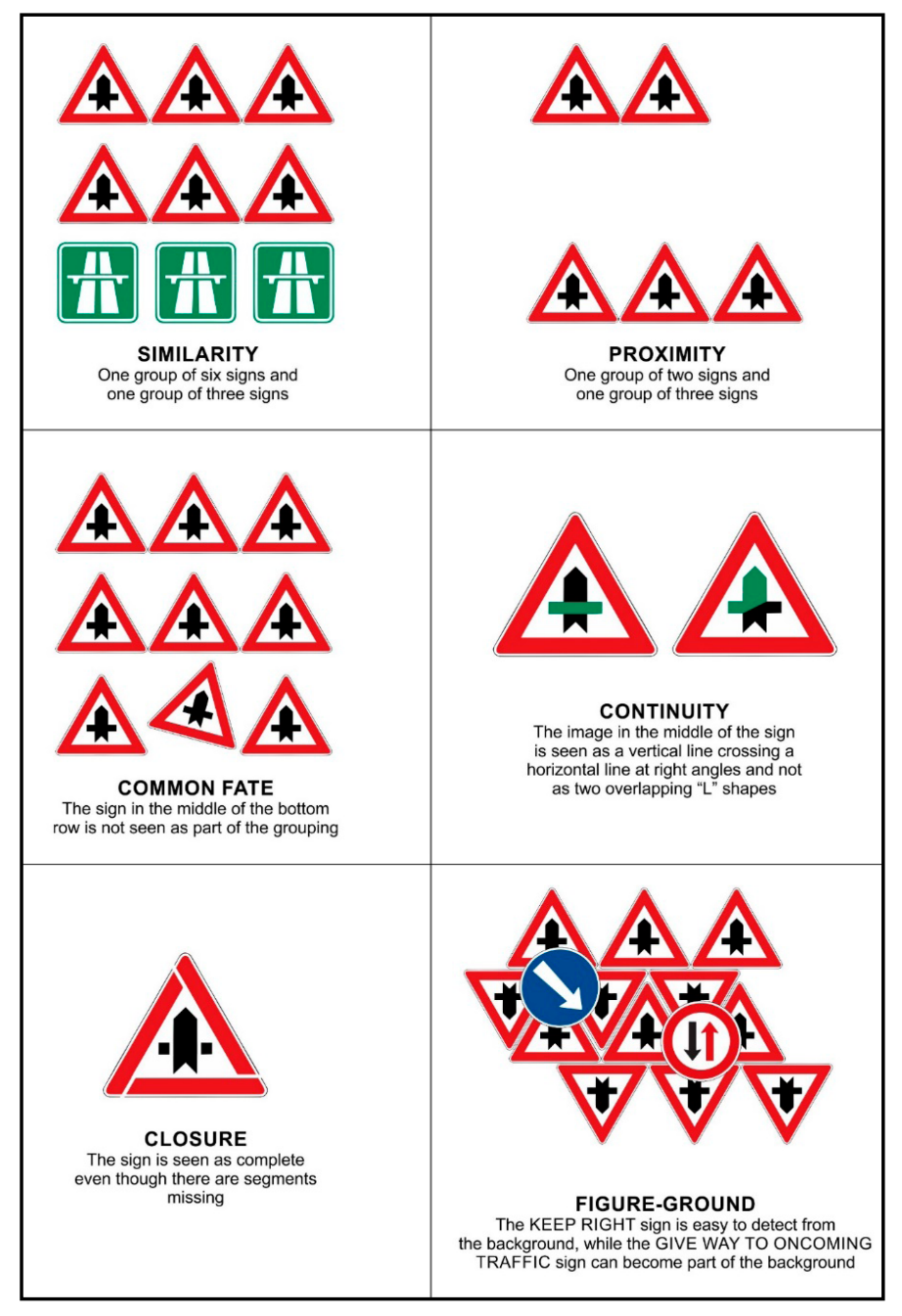

Figure 1: The Gestalt principles of visual grouping

Although not a traditional Gestalt rule, visual perception (especially in driving) can also be heavily influenced by past experiences. Visual stimuli are often grouped together if stimuli have been seen together in the past experience of the observer. This dovetails with the concept of driver expectancy, which has been a long-established tenet of safe road design [22].

Readers looking for a more detailed and comprehensive description of the Gestalt rules and there theoretical foundations are referred elsewhere [23].

The Gestalt rules of perception are descriptive rather than explanatory and generally lack specificity. Nonetheless, these rules are important to know and helpful in designing roads, conducting forensic investigations of traffic crashes, and/or providing a safe road system.

Failure to properly account for the Gestalt laws of visual grouping can lead to a misinterpretation of the visual scene where the brain incorrectly processes the 
visual data it receives (i.e., a perceptual illusion). Perceptual illusions can lead to improper speed and path choices, violate driver expectancies, and increase the chance of crashes. The application of good Gestalt, on the other hand, promotes safe driving.

\section{EXAMPLES OF VISUAL GROUPING IN ROAD SAFETY}

\subsection{Figure-ground}

Figure-ground refers to the relationship between an object and its surround. The concept of figure-ground perception is the one of the most fundamental ways that individuals simplify a visual scene. Distinguishing figures from backgrounds is probabilistic, with road users taking all relevant visual cues into account and making a best-guess at distinguishing figures from backgrounds. When interpreting visual data, everything that is not a figure is ground. A figureground relationship is stable when it is easy to pick out the figure from the ground, and unstable when the figure is not detectable from the ground.

Violations of this Gestalt rule mainly arise in urban areas and are related to complex visual scenes and visual clutter. When the visual scene is replete with different colours and shapes, it can be difficult for the road user to detect traffic signs and signals that are intended to stand out from their environment.

The street scene in Figure $2 \mathrm{a}$, is a busy urban roadway where the traffic signal heads are not easily distinguished from the background of a lavishly painted railway bridge. Similarly, the road user is strained to separate the traffic sign (figure) from the foliage (ground) when traversing the scenic route in Figure $2 b$.

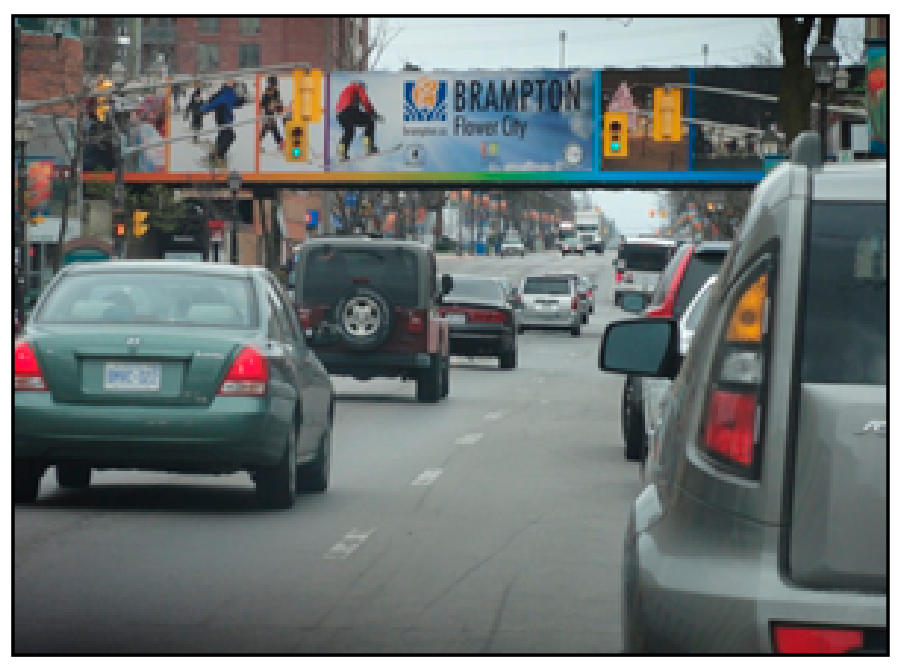

Figure 2a: Complex figure-ground scene in an urban setting

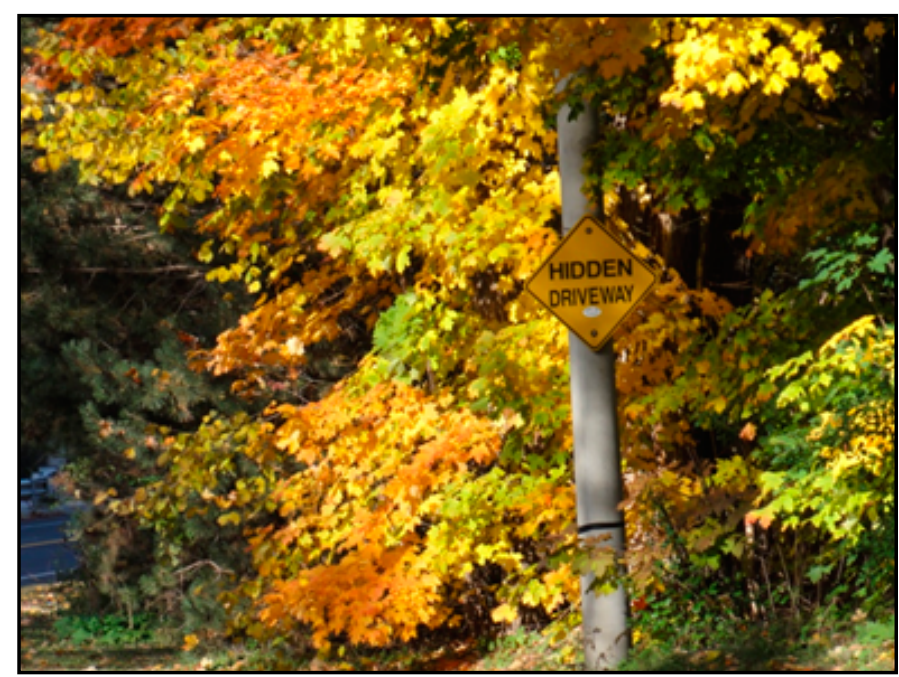

Figure 2b: Complex figure-ground scene in a rural setting

In both instances the traffic control devices are in plain view of the approaching motorist. However, neither device is conspicuous or easily detected from the background. When motorists have trouble distinguishing figures, such as road signs, from their background, then important information can be missed, leading to performance errors and motor vehicle crashes.

As a motorist moves along the roadway, her/his attention shifts to different parts of the visual scene. As attention shifts, the "ground" also shifts - with objects going from figure to ground and back again. It is important in a road safety sense to make objects that are important for safe driving to be clearly identifiable from the ground in order to (attract and) focus attention and minimize perceptual confusion.

\subsection{Similarity}

A good example of visual grouping by similarity is coloured paving that visually separates different areas of the roadway for different uses (or non-use). For example, the aprons of the central islands at roundabouts are often visually distinct from the circulating lanes through the use of coloured paving. Similarly, coloured paving is sometimes used to distinguish exclusive use lanes, like high occupancy vehicles or bicycleonly lanes, from general-purpose traffic lanes. In the above situations, there is no physical restriction from motorists entering the coloured paving area, however, because they are dissimilar to the general-purpose traffic lanes, they are not visually grouped with general-purpose lanes.

Another good example of visual grouping by similarity is the sign array shown in Figure 3. The sign on 
the left guides road users to a toll route, and the road authority partly conveys the message that the route to the left is different from the other route choices by making the sign dissimilar to the other signs through distinct colouring.

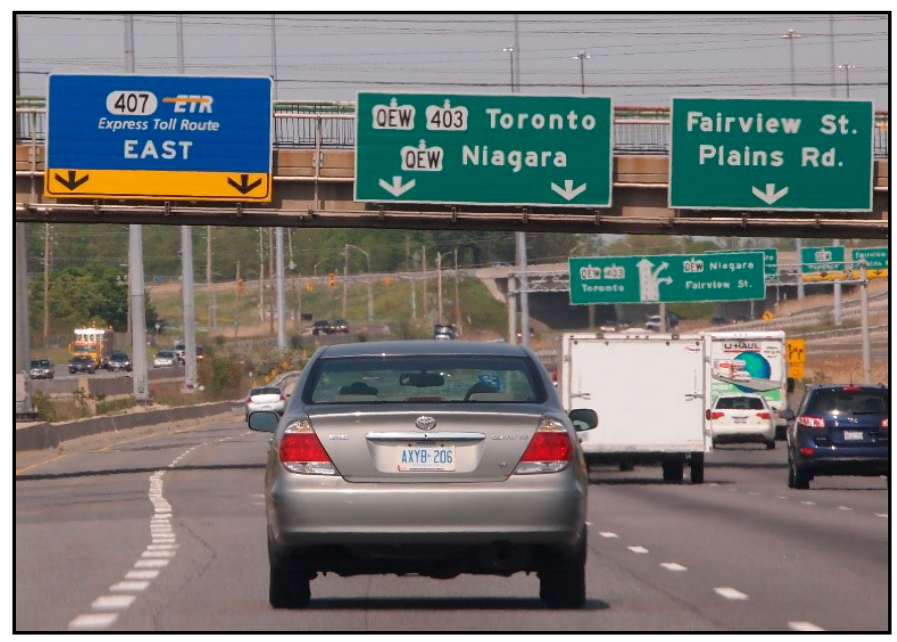

Figure 3: Visual grouping of signs by similar colour

\subsection{Proximity}

The principle of proximity dictates that proximate elements of the roadway will be seen by the motorist as a group or somehow related to one another. Failures in this regard ordinarily result from poor placement of traffic control devices with respect to the geometric features of the road, or to other traffic control devices.

A situation where visual grouping by proximity has created an undue risk of rear-end collisions is shown in Figures 4 . The road authority has erected an overhead guide sign directing westbound traffic to the entrance ramp for a controlled-access highway at the standard distance upstream of the ramp entrance. Although the sign placement conforms to the minimum advance placement requirements, it creates an issue in that the sign is immediately upstream of a side street. The proximity of the sign to the side street, creates a cognitive link between the sign and the street, and motorists frequently turn right onto the side street while attempting to access the controlled-access highway. This performance error is exacerbated by the fact that the actual entrance ramp is primarily hidden from view due to the horizontal alignment of the main road.

Another example where the proximity principle is violated is with poor placement of speed limit signs with respect to other traffic signs. Inappropriate speeds and undue crash risk may result from speed limit signs that are located between reduced-speed warning signs and the related hazard. For example, an $80 \mathrm{~km} / \mathrm{h}$ speed limit sign located downstream of a curve warning sign with a $40 \mathrm{~km} / \mathrm{h}$ advisory speed, and upstream of the physical curve. The $80 \mathrm{~km} / \mathrm{h}$ speed limit sign is more proximate to the actual curve and may be interpreted as an appropriate speed for curve driving.

\subsection{Continuity}

The continuity principle contends that, when an individual is presented with a series of intersecting lines, the lines that are aligned and present a smooth path are perceived as a single continuous line. Abrupt changes in direction are seen as the intersection of two individual elements rather than one element. Individuals have a propensity to see continuous elements as connected and are more apt to follow the continuous line.

The principle of continuity is a consideration in safety-conscious planning. When approaching an intersection with four legs, motorists expect the road on which they are travelling to continue on the opposing leg (usually a straight through movement). Situations where the motorist must turn left or right to stay on the same road are violations of the continuity principle and lead to missed turns, last minute lane changes, abrupt braking, and other unsafe behaviours.

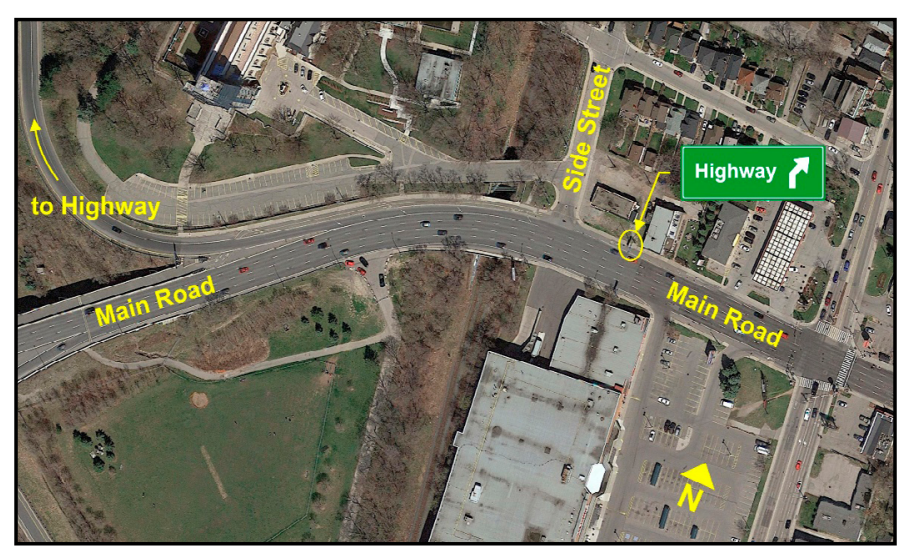

Figure 4: Traffic sign visually grouped with a side street due to proximity

Similarly, a relatively common design where the continuity principle creates problems for motorists is when a private driveway is longitudinally aligned with a public road that terminates at the driveway (Figure 5). Even if the private access is materially different with respect to road design, the alignment 
creates continuity, and may cause motorists to continue at speed down a private access that is not designed to accommodate high speed traffic. In Figure 5, the public road terminates at the STOP sign, but appears to continue on the other side of the intersecting road.

Continuity issues also arise at intersections where a two-way street is opposed by a one-way street where moving traffic is being brought into the intersection. Motorists on the two-way street approaching the intersection may erroneously conclude that the road continues across the intersection and collide head-on with opposing traffic.

\subsection{Closure}

Closure causes individuals to see a visual connection or continuity between sets of elements which do not actually touch each other in a composition. The principle of closure applies, and is particularly troublesome, in conditions of low light when outlines, silhouettes, features and light sources are aligned in such a way that motorists tend to connect the images and see complete figures even when part of the visual information is missing. Motorists use easily recognizable patterns drawn from memory (i.e., past driving experience) and expectancies to fill in missing information and/or make connections between different visual elements, forming a complete image.

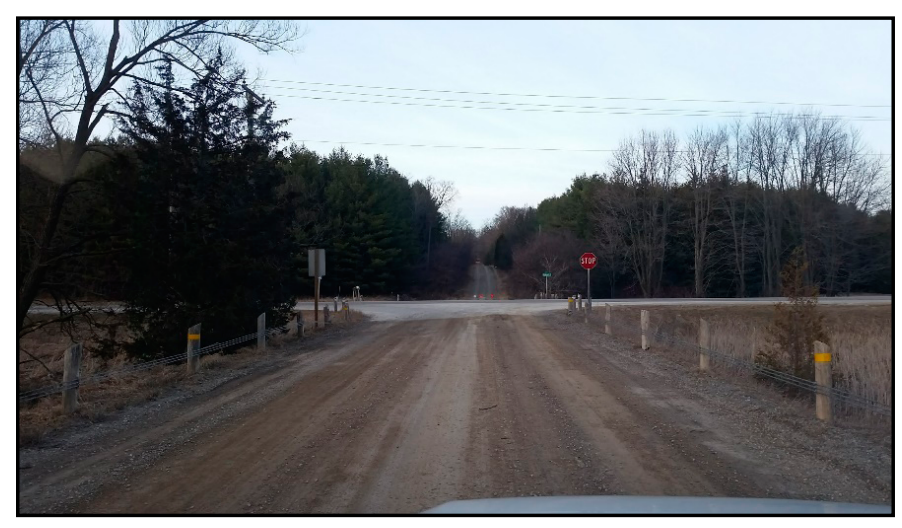

Figure 5: Visual grouping of a driveway to a public road through continuity

The road shown Figure 6 was the site of a serious casualty, night-time crash where a motorist inexplicably drove straight when the road quite obviously curves to the left before the STOP sign. There were no indications that the motorist attempted to steer left, even at the last moment. Upon investigation, it was determined that the subject location presents the motorist with a perceptual illusion or visual trap that becomes apparent in conditions of low light. The propensity for road users to "see" the road as continuing straight is explained by the Gestalt law of closure.

On this unilluminated rural road, during conditions of low light, the visual information available to the motorist regarding roadway alignment includes the short length of road illuminated by the vehicles headlamps, the single street light and the pavement it illuminates on the far side of the intersection, and the headlamps and tail lights of any vehicles on the far side of the intersection. In this situation, the motorist will cognitively connect the road with the street light and the vehicles lights, and assume that the road continues straight, when it actually curves to the left before the intersection. This results in motorists driving off the road to the right since the grassed peninsula in the foreground is not easily detected in dark conditions. The propensity to close the gaps in visual information and assume that the visible elements of the infrastructure are connected results from the principle of closure.

While full illumination of the roadway, to make the actual alignment of the road evident, may be impractical or unaffordable, delineation devices (e.g., chevron alignment signs) placed around the curve would counteract the perceptual grouping.

Closure is also a common visual grouping phenomenon that encourages pedestrians to cross the street where crossing is prohibited. When trails or footpaths exist on both sides of the street are aligned, and there is no crosswalk, pedestrians may visually connect to paths.

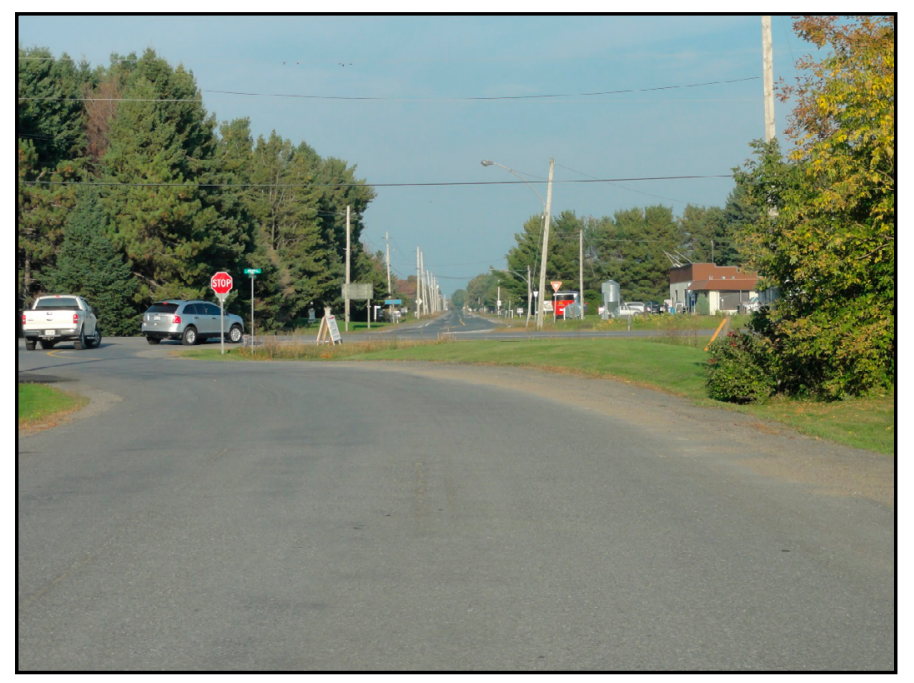

Figure 6: A hazard created by the visual grouping principle of closure 
The likelihood of visual grouping through closure is inversely proportional to the mental effort required to "connect the dots". If visual gaps are small and a recognizable pattern or form is expected closure occurs and motorists easily perceive a completed form. Closure is less likely to occur when the visual gaps are large, or a form is unexpected. The harder it is to make sense of the image the less likely closure will occur.

\subsection{Common fate}

The common fate principle is particularly applicable, although not exclusive to, moving traffic. When operating a vehicle in a platoon of vehicles, or walking within a group of people, those vehicles/individuals that are moving in the same direction as you are related to you by your common direction and destination (i.e., your common fate). Even though this visual grouping may not be conscious, it does occur.

A good example of the common fate principle as it relates to road design is parallel linear systems. Roads are frequently constructed beside and parallel to rail lines, water courses, utility poles, etc. When the road and the adjacent element are parallel for a significant distance the two linear elements become cognitively linked and may be perceived as sharing a common fate. The road safety issue that is caused by this perceptual grouping arises when the two systems diverge. At the diverge road users may inadvertently continue to follow the alignment of the parallel element rather than the road alignment. An example of a diverge is shown in Figure 7. The utility pole line (the red dots), which follows the road alignment for several kilometres continues straight while the road curves right (as one moves southbound). This can lead to motorists continuing straight past the end of the road.

Common fate may also explain what has been termed a sympathetic movement during a protected left-turn phase at a traffic signal. In this situation a motorist who is at the start of a queue and waiting for a green signal indication to proceed straightthrough an intersection will sometimes enter the intersection on a red indication when a left-turn-only arrow is presented. This unintended movement occurs because the through motorist is "grouped" with the other vehicles moving in the same direction and expects to share a common fate (assuming that both are able to move forward) with the adjacent vehicle (in the left-turn lane). As the left-turning motorist initiates the turn on the left-turn phase, the through motorist also moves (mistakenly) risking a crash with an opposing left-turning vehicle.

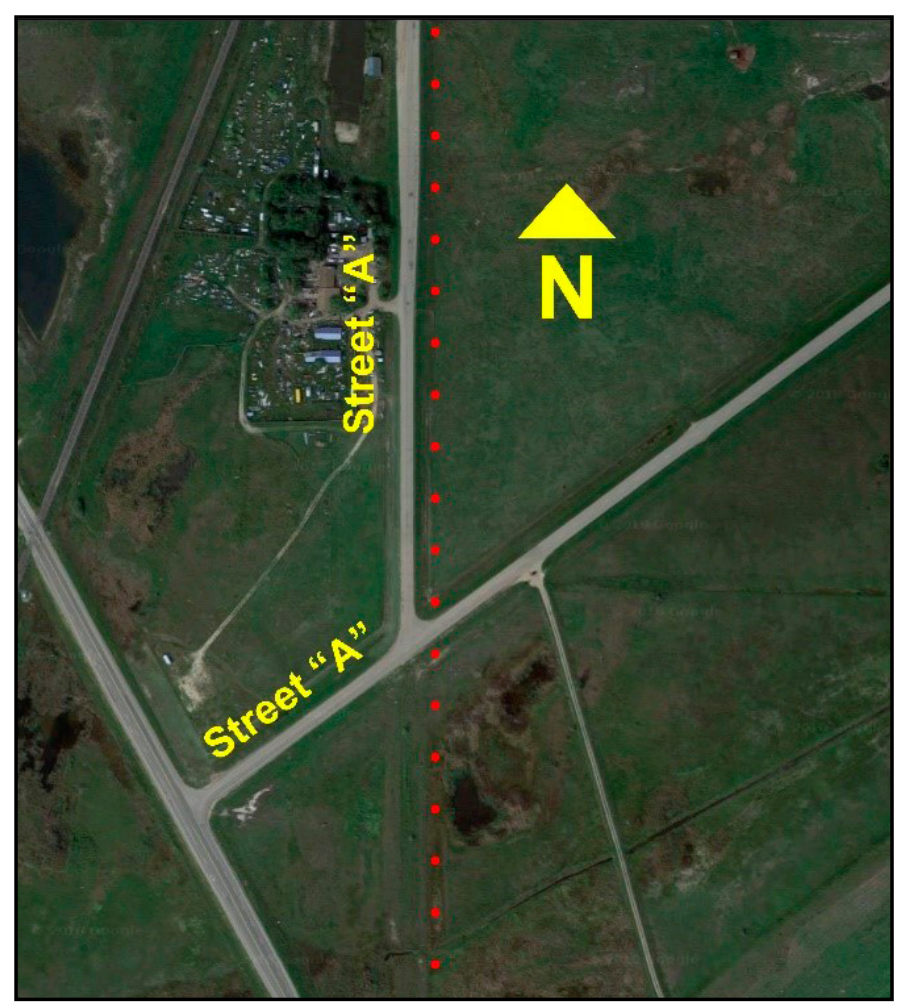

Figure 7: Common fate creating a traffic hazard

\section{DISCUSSION}

When interpreting visual information, the principles of visual grouping create powerful and lasting impressions that guide perception. Because visual grouping is an automatic cognitive process it is difficult to undo grouping without explicit and strong visual information to the contrary. Short of roadway redesign, the "information" needed to counteract the visual grouping is typically delivered via traffic control devices (e.g., signs and delineators) that better inform motorists of the actual roadway alignment. Without these traffic control devices, the perceptual grouping can create an illusion that is a violation of driver expectancy, and a trap for the unwary motorist.

Research in the field of visual grouping has also determined that it is difficult for individuals to ignore irrelevant information that belongs to the same group as task-relevant information [24]. This means that visual grouping has the potential to distract as well as mislead. For example, incorrect placement of route names and/or destinations on a navigation/guide sign may invoke grouping when none is intended, and potentially distract or mislead motorists. 
The Gestalt visual grouping principles described above have stood the test of time and have formed the basis for a substantial amount of modern research on perceptual grouping. Over the years it has become clear that the principles of visual grouping are not absolute, rather they are rules that exist as ceteris paribus. This means each principle applies when the other principles do not apply or are being held constant. When two (or more) principles apply to the same road scene, they may be complementary (i.e., favouring the same grouping) or opposing. In the latter instances, usually one principle is superior and dominates the visual grouping or the organization of the scene is unclear. Several experiments to create a hierarchy among the grouping principles have been undertaken, but an ultimate determination of which principle will dominate in which circumstances remains to be worked out in detail. For example, the road in Figure 8 presents conflicting Gestalt principles of similarity (through pavement type and road width) and continuity (the driveway is longitudinally aligned with the public road).

The knowledge base on perceptual grouping is constantly evolving, with new rules being introduced all the time. Researchers have proposed symmetry as a rule, which asserts that elements that are symmetrical to each other tend to be perceived as a unified group. Situations where a lack of symmetry may become a road safety issue are road sections that proceed through an uneven "cut", staged freeways, and work zones with an uneven number of lanes. In any of these situations, particularly in conditions of low light, or when snow may be obscuring pavement markings, a motorist may be confused as to where the "centre" or directional dividing line of the road is located, increasing the chances of head-on or sideswipe crashes.

Additional rules that have been proposed or explored include, the common region principle [25], where items are seen as a group if they are enclosed within a common boundary, and the connectedness principle [26], where items that are connected by a line are grouped. An example of the common region principle is a traffic signal head where three or more individual signal indications share a common border (the housing of the signal head) and are seen as a group rather than as individual lights.

One of the important factors to be considered when using the rules of visual grouping to assess the relative safety of a location is to consider the appearance of the road in all environmental and lighting

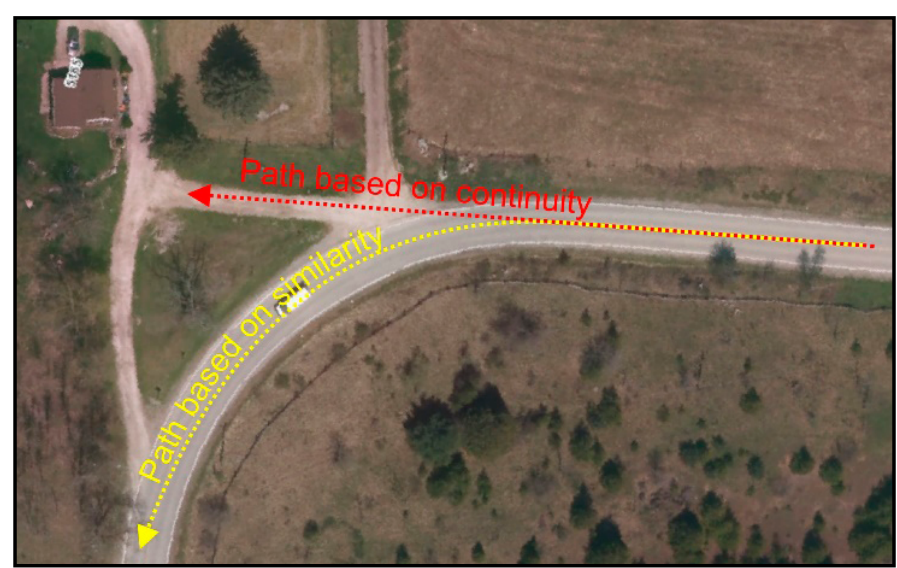

Figure 8: Competing principles of visual grouping

conditions. Visual inputs in conditions of low light can be vastly different from the inputs that are available in full daylight. Similarly, as seen in Figure 2b, the autumn weather changes the otherwise green leaves to yellow, creating difficulty in detecting a yellow warning sign from the background. Studies have also shown that performance in visual search is not constant throughout the day $[27,28,29]$. There is typically a "postlunch" decline in visual search performance, which may also suggest that Gestalt rules of visual grouping play a more important role during this period.

The extent to which visual illusions created by the Gestalt rules of visual grouping contribute to crash occurrence is undetermined. It is likely that these illusions are relatively rare, and they contribute to only a small part of the road safety problem. Further complicating matters is that the impact of the Gestalt rules on road user performance varies between individuals and within individuals, depending on conditions/times. For example, research indicates that familiarity and training impact visual grouping [30]. Prior exposure to a visual illusion may reduce the chances that it will have a negative effect on road user performance. Having stated that, there is a limit to which training can regulate the visual grouping principles, at least in some circumstances [31]. Moreover, the ability to visual group elements develops with age [32], suggesting illusions due to visual grouping may be more of an issue for older road users.

Road safety is predicated on road users making good decisions concerning speed and path for the various road and traffic conditions encountered. Since these decisions are greatly influenced by the visual scene, including the geometry of the road, the traffic control devices present, and the surrounding land use, road designers and traffic engineers should 
be informed about how road users interpret visual inputs. The principles of visual grouping are a fundamental part of understanding how road users will perform on the roadway.

Acknowledgements: The author is grateful to Dr. Robert Dewar for reviewing and providing helpful comments on this paper.

\section{REFERENCES}

1. Treat JS, Tumbas NS, McDonald ST, Shinar D, Hume RD, Mayer RE, Stansifer RL, Castellan NJ (1979) Tri-Level Study of the causes of traffic accidents: Final report, Report No. DOT HS 805 099, May 30, 1979, United States Department of Transportation, National Highway Traffic Safety Administration.

2. Sabey, B. E., \& Taylor, H. (1980). The known risks we run: the highway. In R. C. Schwing \& W. A. Albers Jr (Eds.) Societal risk assessment: how safe is safe enough? Plenum Press, New York.

3. Singh, S. (2015, February). Critical reasons for crashes investigated in the National Motor Vehicle Crash Causation Survey. (Traffic Safety Facts Crash•Stats. Report No. DOT HS 812 115). Washington, DC: National Highway Traffic Safety Administration.

4. Sivak, M (1996). The Information that Drivers Use: Is It Indeed 90\% Visual? Perception, 25, 1080-1089.

5. Booher HR (1978) Effects of visual and auditory impairment in driving performance, Human Factors 20, 307-320.

6. Bryan (1957) Research in vision and traffic safety, Journal of the American Optometric Association, 29, 169-172.

7. Brackstone MA, Waterson BJ (2004) “Are we looking where we are going? An exploratory examination of eye movement in high speed driving" in Proceedings of the $83^{\text {rd }}$ Annual Meeting of the Transportation Research Board, Transportation Research Board, Washington, DC.

8. Mourant RR, Rockwell TH, Rackoff NJ (1969) “Drivers' eye movements and visual workload”, Highway Research Record No. 292, Highway Research Board, Washington, DC.

9. Miller CO (1976) "The Design-Induced Part of the Human Error Problem in Aviation", Journal of Air Law and Commerce 42(1), 119-131.

10. Transportation Association of Canada, Geometric Design Guide for Canadian Roads, Ottawa, ON, Canada (1999)

11. American Association of Highway State Transportation Officials, A Policy on Geometric Design of Highways and Streets, 6th Edition, Washington, DC, 2011

12. Highways Agency, Design Manual for Roads and Bridges: Volume 6 - Road Geometry,

13. PIARC (2016) Human factors guidelines for a safer manroad interface, Report 2016R20EN,
14. Forbes G, Dewar R, Hanscomb F, Alexander G (2013) Applied human factors in road safety guide, Transportation Association of Canada, Ottawa, ON.

15. Campbell JL, Lichty MG, Brown JL, Richard CM, Graving JS, Graham J, O'Laughlin M, Torbic D, and Harwood D (2012) Human factors guidelines for road systems, Second Edition, National Cooperative Highway Research Project Report No. 600, Transportation Research Board, Washington, DC.

16. Kohler W (1923) "Zur Theories des Sukzessivvergleichs und der Zeitfehler”, Psychologische Forschung 4, 115-175.

17. Wertheimer M (1923) "Untersuchungen zur Lehre von Gestalt, II”, Psychologische Forschung 4, 301-350.

18. Heider GM (1977) "More about Hull and Koffka”, American Psychologist, 32, 383.

19. Weller G, Dietze M "SER and SER Approaches: State-ofthe-art”, Report No. WP01-01 of ERASER - Evaluation to Realise a Common Approach to Self-explaining, June 2010.

20. Weller G, Schlag B, Friedel T, Rammin C “Behaviourally relevant road categorisation: A step towards self-explaining rural roads", Accident Analysis \& Prevention 40 (2008), Elsevier, 1581-1588, doi:10.1016/j.aap.2008.04.009.

21. Herrstedt L "Road User Ability and Behaviour - The Basis for a Safe and Road User Friendly Road Design”, $4^{\text {th }}$ International Symposium on Highway Geometric Design, June 2010.

22. Alexander GJ, Lunenfeld H, "Driver Expectancy in Highway Design and Traffic Operations", Federal Highway Administration, Report No. FHWA-TO-86-1, Washington, DC, April 1986.

23. Wagemans J, Elder JH, Kubovy M, Palmer SE, Peterson MA, Singh M, von der Heydt R (2012) "A Century of Gestalt Psychology in Visual Perception I: Perceptual Grouping and Figure-Ground Orgnization”, Psychological Bulletin, 138(6), 1172-1217.

24. Driver J, Baylis G (1989) "Movement and visual attention: The spotlight metaphor breaks down”, Journal of Experimental Psychology: Human Perception and Performance, 17, 561-570.

25. Palmer S (1992) "Common region: a new principle of perceptual grouping”, Cognitive Psychology, 24, 436-447.

26. Palmer S, Rock I (1994) "Rethinking perceptual organization: The role of uniform connectedness", Psychonomic Bulletin \& Review, 1, 29-35.

27. Blake (1967) "Time of day effects on performance in a range of tasks", Psychonomic Science, 9, 349-350.

28. De Gennaro L, Ferrara M, Curcio G, Bertini M (2001) "Visual search performance across $40 \mathrm{~h}$ of continuous wakefulness: Measures of speed and accuracy and relation with oculomotor performance”, Psysiology \& Behavior, 74, 197-204.

29. McDougall S, Tyrer V, Folkard S (2006) "Searching for Signs, Symbols, and Icons: Effects of Time of Day, Visual Complexity, and Grouping", Journal of Experimental Psychology: Applied, 12(2), 118-128. 
30. X. Vecera SP, Farah MJ (1997) "Is image segmentation a bottom-up or an interactive process?", Perception and Psychophysics, 59, 1280-1296.Kimchi R, Hadad B (2002)

"Influence of past experience on perceptual grouping", Psychological Science, 13(1): 41-47.

31. Goldstone RL (2000) "Unitization during category learning”, Journal of Experimental Psychology: Human Perception and Performance, 26(1): 86-112.

32. Kovacs I (2000) "Human development of perceptual organization”, Vision Research, 40(10-12):1301-1310. 